\title{
Processing-Microstructure Relationships From 3D Characterization of Additively Manufactured Metals
}

Andrew T. Polonsky ${ }^{1 *}$, Narendran Raghavan ${ }^{2}$, McLean P. Echlin ${ }^{1}$, William C. Lenthe ${ }^{3}$, Michael M. Kirka $^{2}$, Ryan R. Dehoff ${ }^{2}$, and Tresa M. Pollock ${ }^{1}$

1. Materials Department, University of California Santa Barbara, Santa Barbara, CA, USA.

2. Oak Ridge National Laboratory, Oak Ridge, TN, USA.

3. Department of Materials Science and Engineering, Carnegie Mellon University, Pittsburgh, PA, USA.

* Corresponding author: polonsky@ucsb.edu

Additive manufacturing (AM) has received renewed interest over the past several years as a small-tomedium-scale manufacturing process. No longer a rapid prototyping technique, the layer-by-layer fabrication of metallic systems offers unprecedented design flexibility for geometrically complex, enduse components [1]. However, the application of AM parts is currently limited due to variable mechanical properties, commonly linked to heterogeneity in the microstructure and defects created during processing $[2,3]$. Control of the heat source to adjust thermal conditions has been demonstrated for microstructural control in AM components on the bulk scale $[4,5]$.

To fully realize the potential of the processing space offered by AM techniques and improve the reliability of AM parts, a more comprehensive model of processing-microstructure relationships is required to control microstructure on the scale of individual melt pools. Here, we present datasets collected from several AM samples via TriBeam tomography [6]. These 3D volumes, on the order of a cubic millimeter, investigate the solidification phenomena controlling microstructure at the melt pool scale.

Samples of nickel-base alloy Inconel 718 were fabricated using Electron Beam Melting (EBM), a powder bed AM technique. Using variable beam settings, isolated melt pools were deposited onto blocks built with distinct bulk microstructures. Serial sectioning of these isolated melt pools and the surrounding bulk was performed in the TriBeam system to obtain crystallographic and microstructural data. One melt volume, deposited onto a highly-textured, coarse-grained microstructure, was investigated to characterize the columnar-to-equiaxed transition occurring at thermal conditions relevant to AM processing. A 3D reconstruction of the volume is shown in Figure 1. Experimental results were compared to TRUCHAS solidification simulations to calibrate the models used to inform processing parameters. Another melt volume deposited onto a weakly textured microstructure was used to characterize the development of bulk microstructure from a collection of finely-spaced, individual spot melts. The connectivity information afforded by $3 \mathrm{D}$ characterization allows for the investigation of competitive grain growth on scales relevant to AM processes.

A volume of 304L stainless steel fabricated using Laser Engineered Net Shaping (LENS), a blown powder process, was also collected in the TriBeam system to investigate the origin of the complex grain morphologies and highly anisotropic microstructures commonly observed in AM samples. Characterization of a melt volume from the bulk provides insight into the formation of long-range orientation gradients in excess of $10^{\circ}$ within individual grains. One such large grain comprising roughly one-fifth of the collected volume is shown in Figure 2. Orientation gradients have been correlated to the path of the solid-liquid interface during solidification, and the role of chemical segregation provides a potential approach for AM-specific alloy design [7]. 


\section{References:}

[1] WJ Sames et al., International Materials Reviews 61 (2016), p. 315-360.

[2] JJ Lewandowski and M Seifi, Annual Review of Materials Research 46 (2016), p. 151-186.

[3] AT Polonsky et al., Materials Characterization 143 (2018), p. 171-181.

[4] RR Dehoff et al., Materials Science and Technology 31 (2015), p. 931-938.

[5] N Raghavan et al., Acta Materialia 112 (2016), p. 303-314.

[6] MP Echlin et al., Review of Scientific Instruments 83 (2012), p. 023701.

[7] The authors acknowledge funding support from Oak Ridge National Laboratory under Award No. 400156470 .

(a)

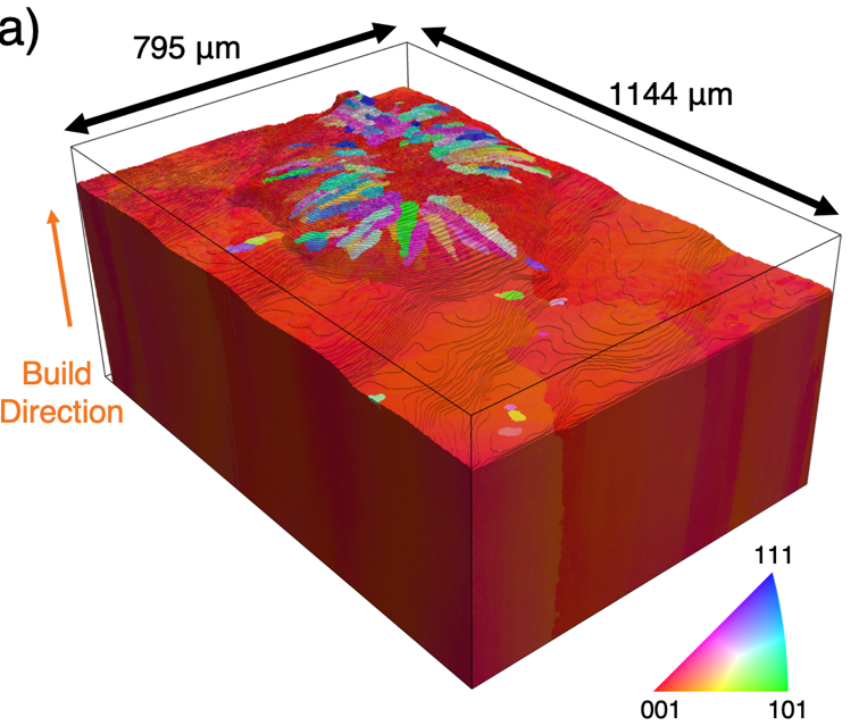

(b)

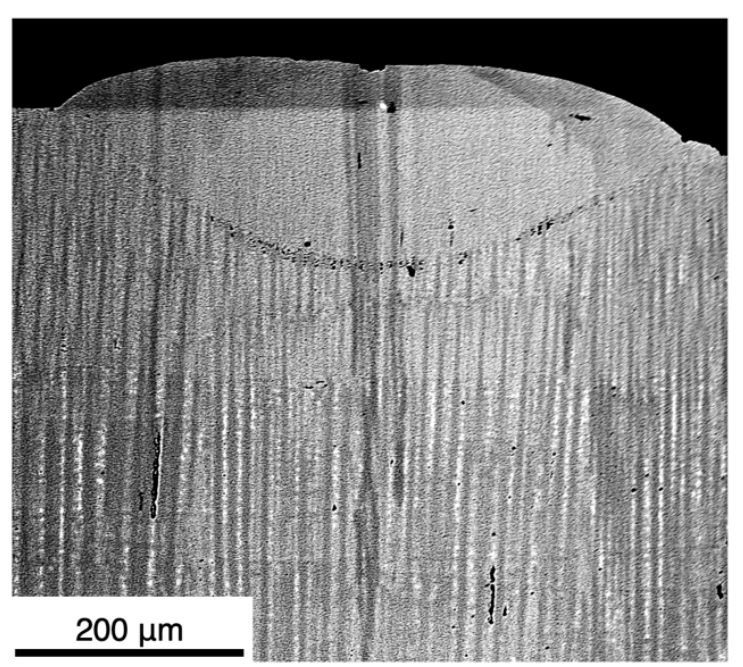

Figure 1. A 3D dataset of Inconel 718 collected around an isolated melt pool of an EBM sample (a). The melt pool was extracted using backscatter electron images (b) taken throughout the volume.

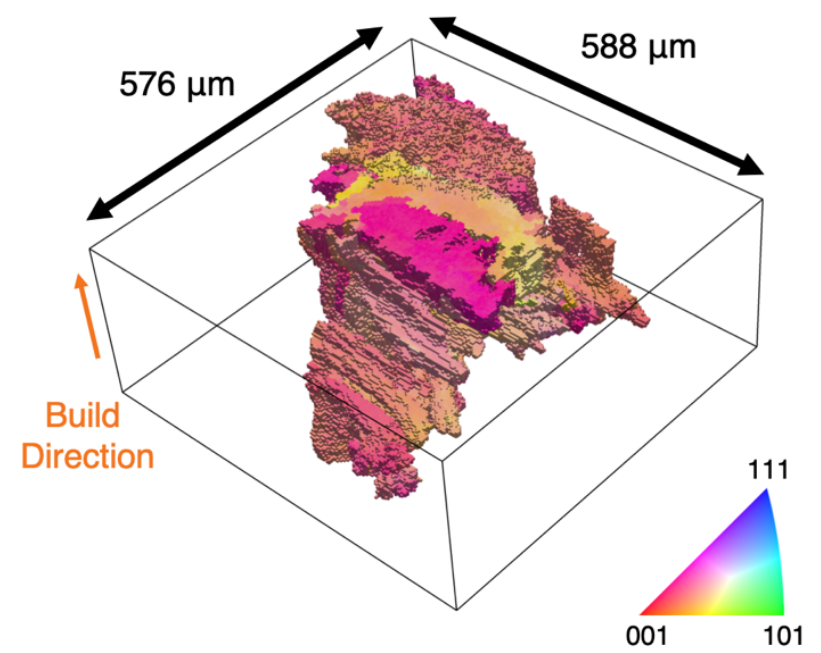

Figure 2. A large grain from a sample of 304L stainless steel fabricated using the LENS process. The complex grain morphology and large misorientation gradients, visible as changes in inverse pole figure coloring, make this class of features difficult to characterize with conventional 2D microscopy. 